\title{
COGNITIVE TESTS USED IN EXAMINING EFFECTS OF PHYSICAL TRAINING IN ELDERLY PEOPLE
}

\section{TESTY POZNAWCZE STOSOWANE W BADANIU EFEKTÓW TRENINGU FIZYCZNEGO U OSÓB STARSZYCH}

\author{
${ }^{1}$ Cognitive Science, Humanities Faculty, Nicolaus Copernicus University in Toruń \\ ${ }^{2}$ Department of Geriatrics, Collegium Medicum in Bydgoszcz, Nicolaus Copernicus University in Toruń
}

\section{S u m m a r y}

Introduction. Cognitive tests are a valuable, sensitive and useful tool for examining the effectiveness of physical training.

Purpose. To show which studies are sensitive in examining cognitive changes induced by physical training. We explored meta-analysis with healthy participants and patients with MCI.

Materials and methods. Analysis of metaanalysis in the EBSCO database using keywords: metaanalysis, cognitive tests, physical training, elderly.
Results. 4 meta-analysis have 10 researches in common; however, diverse of inclusion and exclusion criteria led to inability in obtaining reliable results.

Discussion and conclusion. Some cognitive tests seems to be more sensitive in examining results of physical training. Diversity of research methodology induces much difficulties in comparing of studies.

\section{Streszczenie}

W s t ę p . Testy poznawcze są wrażliwym i użytecznym narzędziem w badaniu skuteczności treningu fizycznego.

C e 1. Sprawdzenie, które testy są wrażliwe na badania zmian poznawczych wywołanych przez trening fizyczny. Przeanalizowaliśmy metaanalizy ze zdrowymi uczestnikami i pacjentami z MCI.

Materiał i metody. Analiza metaanaliz w bazie danych EBSCO za pomocą słów kluczowych: metaanaliza, testy poznawcze, ćwiczenia fizyczne, osoby starsze.
Wyniki. 4 metaanalizy posiadają 10 wspólnych badań, jednakże zróżnicowanie kryteriów włączenia i wyłączenia doprowadziły do niemożności w uzyskaniu wiarygodnych wyników.

Dyskusja i wnioski. Niektóre testy poznawcze wydają się być bardziej czułe w badaniu wyników treningu fizycznego. Różnorodność metodologii wywołuje wiele trudności w porównywaniu badań. 


\section{INTRODUCTION}

There are several factors which influence cognitive maintenance in the years of senile: subjects' sex, years of education, economic situation, overall state of health, years of physical exercising. Impact of physical training on cognitive functioning of the elderly has been reported multiple times for the past 3 decades $[1,2]$. Moreover, meta-analysis from last decade confirmed positive effect of physical training on cognitive functioning [3-6].

However, underlying neurobiological mechanism is still unknown. Enhanced cardiovascular functioning is one of the hypothesis, widely described in other reviews $[3,7,8]$.

Our aim is to present cognitive tests which were used in examining effects of intervention. First part of our review concerns interventions in Mild Cognitive Impairment (MCI) of the elderly, second one refers to healthy elderly participants.

\section{MATERIALS AND METHODS}

Cognitive tests used in meta-analyzes: effect of physical training on cognitive functioning in elderlies with MCI and dementia

Patricia Heyn et al. [4] meta-analysis is based on 30 researches containing MMSE (Mini-Mental State Examination). Researches included in meta-analysis examined effect of physical training in patients with MCI or dementia. Interestingly, MCI as a group of clinical syndromes has multiple diagnostic criteria [9]. Participants' MMSE average scores varied from 6 to 25 points [4]. It shows difference in pre-test overall health state of participants from interventions included in meta-analysis, what underlies problems in comparing size of effects in particular researches.

Gates et al. [3] meta-analysis included fourteen random control trials with MCI participants. Only $8 \%$ of cognitive outcomes were statistically significant. Cognitive Participants' MMSE scores ranged from 24 to 28 points, age varied between 65 to 95 years. Interestingly, Gates et al. [3] shows no effects of aerobic training in memory improvement in the analyzed researches. Effects induced by aforementioned training modality were restricted to verbal fluency only [3]. However, two researches reported large significant effects of strength training on memory improvement [3].
Table I. Noteworthy, a prior meta-analysis reported greatest improvement in researches with groups restricted to no more than 10 participants (modified version of Gates et al. [3])

\begin{tabular}{|c|c|c|c|} 
Test & $\begin{array}{c}\text { Total nr of } \\
\text { intervention } \\
\text { group } \\
240\end{array}$ & $\begin{array}{c}\text { Total nr } \\
\text { of control } \\
\text { group } \\
204\end{array}$ & $\begin{array}{c}\text { Mean Difference IV, } \\
\text { Random, 95\% CI }\end{array}$ \\
\hline Stroop & 394 & 431 & $6.76[-1.19,7.14]$ \\
\hline TMTB & 461 & 484 & $1.32[0.38,2.26]$ \\
$\begin{array}{c}\text { Fluency } \\
\text { Digit Symbol }\end{array}$ & 361 & 331 & $0.57[-1.21,2.34]$ \\
Substitution Test & 299 & 321 & $0.15[-0.12,0.42]$ \\
\hline $\begin{array}{c}\text { Digit Span } \\
\text { Learning/immediate } \\
\text { memory \& delayed } \\
\text { memory }\end{array}$ & 339 & 383 & $-0.01[-0.16,0.14]$ \\
\hline
\end{tabular}

Cognitive tests used in meta-analysis: effect of physical training on cognitive functioning in healthy elderly people

Colcombe and Kramer [5] included eighteen interventions in their meta-analysis. Exclusion criteria were: cross-sectional design, not random assignment, unsupervised intervention, aerobic exercises not included in intervention, age of participants under 55. Colcombe and Kramer analyzed one intervention with MCI participants and one with intervention group suffering from depression. Meta-analysis confirms that physical exercise is beneficial for all analyzed cognitive functions, especially for executive one [5].

Table II. Noteworthy, summed results from 101 participants showed statistically significant difference of effect size between improvement of combined strength and aerobic training group and aerobic training only group (0.59 vs. 0.41, SE=0.043) (adapted from Colcombe and Kramer [5])

\section{Cognitive tests}

executive (Erickson flanker task) controlled (choice reaction time task) spatial (Benton Visual Retention Task) speed (simple reaction time, finger tapping speed)

Angevaren et al. [6] used criteria list for quality assessment of non-pharmaceutical trials (CLEAR NPT). Exclusion criteria refers to, inter alia, researches including depressed participants or in average age under 55, non-randomized trials, lack of fitness parameter or no quantitative data. 11 randomized studies were included into analysis. Cognitive tests used in the analyzed papers were categorized into subcategories according to concept of Lezak $[6,10,11]$.

Angevaren et al. [6] reported that eight of 11 analyzed studies showed that aerobic training influence 
positively on increment in $\mathrm{VO}_{2} \max$ (approx. $14 \%$ ). Increment coincided with better cognitive functioning, with largest effect on motor functioning (effect size 1.17), auditory attention (e.s. 0.50), cognitive speed and visual attention (both e. s. 0.26). Noteworthy, meta-analysis reported 33 cognitive tests only. Several tests were missed in analysis to avoid multiple representation of studies over the categories, and to facilitate the summing of particular results [6].

\section{Comparison 1. Aerobic exercise vs. any intervention}

Table III. 'Any intervention' could be consists of strength training, flexibility training or passive attendance in gym were applied. Comparison between aerobic training and no intervention could result in misleading results. In intervention group, factors other than aerobic training per se, can influence on cognitive functioning improvement (adapted from Angevaren et al. [6])

\begin{tabular}{|c|c|c|c|c|}
\hline Outcome or subgroup title & $\begin{array}{l}\text { No. of } \\
\text { studies }\end{array}$ & $\begin{array}{l}\text { No. of } \\
\text { partici- } \\
\text { pants }\end{array}$ & Effect size & \multirow{9}{*}{$\begin{array}{l}\text { Std. Mean Difference } \\
\text { (IV, Random, 95\% } \\
\text { CI) }\end{array}$} \\
\hline 1 Cognitive speed & 6 & 312 & $0.24[0.01,0.46]$ & \\
\hline 1.1 Simple reaction time & 1 & 37 & $-0.10[-0.75,0.54]$ & \\
\hline 1.2 Trailmaking part A 1 & 1 & 48 & $0.52[-0.06,1.10]$ & \\
\hline 1.3 Digit symbol substitution & 4 & 227 & $0.23[-0.03,0.50]$ & \\
\hline $\begin{array}{l}2 \text { Verbal memory functions } \\
\text { (immediate) }\end{array}$ & 4 & 209 & $0.17[-0.10,0.44]$ & \\
\hline 2.2 Randt Memory test story recall & 2 & 65 & $0.33[-0.16,0.82]$ & \\
\hline $\begin{array}{l}\text { 2.3 Ross Information: Processing } \\
\text { Assessment immediate memory }\end{array}$ & 1 & 20 & $0.06[-0.82,0.93]$ & \\
\hline $\begin{array}{l}\text { 2.4 Rey auditory verbal learning trial } \\
\text { I-V }\end{array}$ & 1 & 124 & $0.10[-0.25,0.45]$ & \\
\hline $\begin{array}{l}3 \text { Visual memory functions } \\
\text { (immediate) }\end{array}$ & 2 & 65 & $0.04[-1.66,1.75]$ & \multirow{14}{*}{$\begin{array}{l}\text { Mean Difference } \\
\text { (IV, Fixed, 95\% CI) } \\
\text { Mean Difference } \\
\text { (IV, Random, 95\% } \\
\text { CI) } \\
\text { Mean Difference } \\
\text { (IV, Fixed, 95\% CI) }\end{array}$} \\
\hline 3.1 Benton visual retention & 2 & 65 & $0.04[-1.66,1.75]$ & \\
\hline 4 Working memory & 3 & 189 & $0.36[-0.31,1.03]$ & \\
\hline 4.1 Digit span backward & 3 & 189 & $0.36[-0.31,1.03]$ & \\
\hline 5 Memory functions (delayed) & 1 & 124 & $0.06[-0.44,1.44]$ & \\
\hline $\begin{array}{l}5.1 \text { Rey auditory verbal learning } \\
\text { delayed recall trial }\end{array}$ & 1 & 124 & $0.06[-0.44,1.44]$ & \\
\hline 6 Executive functions & 7 & 326 & $0.16[-0.20,0.51]$ & \\
\hline 6.1 Trail making part B & 2 & 65 & $0.35[-0.14,0.85]$ & \\
\hline $\begin{array}{l}\text { 6.2 Ross Information: Processing } \\
\text { Assessment problem solving }\end{array}$ & 1 & 20 & $-0.88[-1.81,0.05]$ & \\
\hline $\begin{array}{l}6.3 \text { Wechsler Memory Scales } \\
\text { mental control }\end{array}$ & 1 & 16 & $-0.44[-1.44,0.55]$ & \\
\hline 6.4 Word comparison & 1 & 53 & $0.24[-0.30,0.78]$ & \\
\hline $\begin{array}{l}6.5 \text { Task switching paradigm } \\
\text { (accuracy) }\end{array}$ & 1 & 124 & $0.03[-0.32,0.38]$ & \\
\hline 6.6 Verbal fluency & 1 & 48 & $0.87[0.28,1.47]$ & \\
\hline 7 Perception & 3 & 160 & $-0.10[-0.63,0.43]$ & \\
\hline 7.1 Face recognition (delayed recall) & 1 & 124 & $0.17[-0.18,0.53]$ & \multirow{11}{*}{$\begin{array}{l}\text { Std. Mean Difference } \\
\text { (IV, Random, 95\% } \\
\text { (I) }\end{array}$} \\
\hline $\begin{array}{l}\text { 7.2 Ross Information: Processing } \\
\text { Assessment auditory processing }\end{array}$ & 1 & 20 & $-0.17[-1.05,0.71]$ & \\
\hline $\begin{array}{l}7.3 \text { Wechsler Adult: Intelligence } \\
\text { Scales visual reproduction }\end{array}$ & 1 & 16 & $-0.81[-1.84,0.22]$ & \\
\hline 8 Cognitive inhibition & 3 & 189 & $-0.02[-0.31,0.26]$ & \\
\hline 8.1 Stroop color word (interference) & 2 & 65 & $-0.07[-0.56,0.42]$ & \\
\hline 8.2 Stopping task (accuracy & 1 & 124 & $0.01[-0.35,0.36]$ & \\
\hline 9 Visual attention & 5 & 290 & $0.26[0.02,0.49]$ & \\
\hline 9.1 Digit vigilance & 1 & 48 & $0.45[-0.13,1.02]$ & \\
\hline $9.22 \& 7$ test & 2 & 65 & $0.30[-0.19,0.79]$ & \\
\hline 9.3 Letter search primary task RT & 1 & 53 & $0.05[-0.49,0.59]$ & \\
\hline 9.4 Visual search (accuracy) & 1 & 124 & $0.25[-0.10,0.60]$ & \\
\hline 10 Auditory attention & 5 & 243 & $0.05[-0.45,0.54]$ & \multirow{5}{*}{$\begin{array}{l}\text { Mean Difference } \\
\text { (IV, Random, 95\% } \\
\text { CI) } \\
\text { Std. Mean Difference } \\
\text { (IV, Random, 95\% } \\
\text { CI) }\end{array}$} \\
\hline 10.1 Digit span forward & 5 & 243 & $0.05[-0.45,0.54]$ & \\
\hline 11 Motor function & 4 & 237 & $0.52[-0.25,1.30]$ & \\
\hline 11.1 Finger tapping & 3 & 113 & $0.72[-0.35,1.78]$ & \\
\hline $\begin{array}{l}\text { 11.2 Pursuit rotor task (tracking } \\
\text { error) }\end{array}$ & 1 & 124 & $0.02[-0.33,0.38]$ & \\
\hline
\end{tabular}

Comparison 2. Aerobic training vs flexibility/balance training

Table IV. Flexibility and balance are categorized into one group, nonetheless it resulted in a very few number of studies in each cognitive category (adapted from Angevaren et al. [6])

\begin{tabular}{|c|c|c|c|c|}
\hline Outcome or subgroup title & $\begin{array}{l}\text { No. of } \\
\text { studies }\end{array}$ & $\begin{array}{c}\text { No. of } \\
\text { partici- } \\
\text { pants }\end{array}$ & Effect size & Statistical method \\
\hline 1 Cognitive speed & 3 & 189 & $1.29[-0.41,2.98]$ & Mean Difference \\
\hline 1.1 Digit symbol substitution & 3 & 189 & $1.29[-0.41,2.98]$ & $\begin{array}{l}\text { (IV, Random, 95\% } \\
\text { CI) }\end{array}$ \\
\hline $\begin{array}{l}2 \text { Verbal memory functions } \\
\text { (immediate) }\end{array}$ & 3 & 189 & $0.18[-0.11,0.47]$ & Std. Mean \\
\hline 2.1 Randt Memory test story recall & 2 & 65 & $0.33[-0.16,0.82]$ & $\begin{array}{l}\text { Difference } \\
\text { IV, Random, 95\% }\end{array}$ \\
\hline $\begin{array}{l}2.2 \text { Rey auditory verbal learning trial I- } \\
\text { V }\end{array}$ & 1 & 124 & $0.10[-0.25,0.45]$ & \\
\hline $\begin{array}{l}3 \text { Visual memory functions } \\
\text { (immediate) }\end{array}$ & 2 & 65 & $0.04[-1.66,1.75]$ & \\
\hline 3.1 Benton visual retention & 2 & 65 & $0.04[-1.66,1.75]$ & \\
\hline 4 Working memory & 3 & 189 & $0.36[-$ & \\
\hline 4.1 Digit span backward & 3 & 189 & $0.36[-0.31,1.03]$ & $\begin{array}{l}\text { IV, Ra } \\
\text { CI) }\end{array}$ \\
\hline Mem & 1 & 124 & $0.06[-0.44,1.44]$ & \\
\hline $\begin{array}{l}5.1 \text { Rey } \\
\text { delayed }\end{array}$ & 1 & 124 & $0.06[-0.44,1.44]$ & \\
\hline $6 \mathrm{Exe}$ & 4 & 242 & $0.16[-0$ & \\
\hline $1 \mathrm{Tr}$ & 2 & 65 & & \\
\hline 6.2 Word co & 1 & 53 & $0.24[-0.30,0.78]$ & $\begin{array}{l}\text { Difference } \\
\text { (IV, Random, 95\% }\end{array}$ \\
\hline $\begin{array}{l}6.3 \text { Task switching paradigm } \\
\text { (accuracy) }\end{array}$ & 1 & 124 & $0.03[-0.32,0.38]$ & $\begin{array}{l}\text { (Iv) } \\
\text { (v) }\end{array}$ \\
\hline 7 Per & 1 & 124 & 3.70 & Mes \\
\hline $7.1 \mathrm{~F}$ & 1 & 124 & 08] & (IV, Fixed, 95\% Cl) \\
\hline 8 & 3 & 189 & -0.02 & \\
\hline & 2 & 65 & $-0.07[-0.56,0.42]$ & \\
\hline $\begin{array}{l}\text { 8.2 Stopping task (accuracy choice } \\
\text { RT) }\end{array}$ & 1 & 124 & 0.01 & $\begin{array}{l}\text { Std. Mean } \\
\text { Difference }\end{array}$ \\
\hline Visual attention & 4 & 242 & 0 & IV, Random, 95\% \\
\hline & 2 & 65 & & CI) \\
\hline sk RT & 1 & 5 & & \\
\hline & 1 & 12 & & \\
\hline 10 Auditory attention & 3 & 189 & $-0.20[-0.81,0.40]$ & Mean Difference \\
\hline 10.1 Digit span forward & 3 & 189 & $81,0.40]$ & (IV, R \\
\hline & 3 & 189 & & \\
\hline 11.1 Finger tapping & 2 & 65 & $0.17[-0.31,0.66]$ & Dift \\
\hline 11.2 Pursuit rotor task & 1 & 124 & $0.02[-0.33,0.38]$ & (IV, Random, 95\% \\
\hline
\end{tabular}

\section{Comparison 4. Aerobic exercise vs. strength program}

Table V. The aim of meta-analysis were examining influence of aerobic training on cognitive functioning, however several analyzed studies contained strength intervention as well [6]. Interestingly, according to Gates et al. [3], strength training showed effect size in memory functioning as well (adapted from Angevaren et al. [6])

\begin{tabular}{|c|c|c|c|c|}
\hline Outcome or subgroup title & $\begin{array}{l}\text { No. of } \\
\text { studies }\end{array}$ & $\begin{array}{l}\text { No. of } \\
\text { partii- } \\
\text { cipants }\end{array}$ & Effect size & $\begin{array}{c}\text { Statistical } \\
\text { method }\end{array}$ \\
\hline $\begin{array}{l}1 \text { Verbal memory functions } \\
\text { (immediate) }\end{array}$ & 1 & 20 & $0.30[-4.17,4.77]$ & \multirow{6}{*}{$\begin{array}{l}\text { Mean Difference } \\
\text { (IV, Fixed, 95\% } \\
\text { CI) }\end{array}$} \\
\hline $\begin{array}{l}\text { 1.1 Ross Information } \\
\text { Processing Assessment } \\
\text { immediate memory }\end{array}$ & 1 & 20 & $0.30[-4.17,4.77]$ & \\
\hline 2 Executive functions & 1 & 20 & $-2.30[-4.49,-0.11]$ & \\
\hline $\begin{array}{l}\text { 2.1 Ross Information } \\
\text { Processing Assessment problem } \\
\text { solving and abstract reasoning }\end{array}$ & 1 & 20 & $-2.30[-4.49,-0.11]$ & \\
\hline 3 Perception & 1 & 20 & $-0.06[-2.93,1.93]$ & \\
\hline $\begin{array}{l}\text { 3.1 Ross Information } \\
\text { Processing Assessment auditory } \\
\text { processing }\end{array}$ & 1 & 20 & $-0.06[-2.93,1.93]$ & \\
\hline
\end{tabular}




\section{Common researches included in meta-analysis}

Meta-analysis included in our paper [3-6] consist of 73 analyzed trials. Interestingly, several common interventions were included: 10 researches [1, 12-21] are analyzed in more than one meta-analysis.

Table VI. Common researches causes inability in obtaining reliable results from overall results of analyzed meta-analysis [3-6]

\begin{tabular}{|l|l|l|l|l|}
\hline & Gates et al. [3] & Hayne et al. [4] & Angevaren [6] & Colcombe [5] \\
\hline Gates et al. [3] & & Molloy et al. [12] & & \\
\hline Hayne et al. [4] & Molloy et al. & & & $\begin{array}{l}\text { Dustman et al. [1], } \\
\text { Hill et al. [13], } \\
\text { Perri et al. [14], } \\
\text { Hassmen et al. } \\
\text { [21], } \\
\text { Barry et al. [19], } \\
\text { Powell et al. [20]. }\end{array}$ \\
\hline Angevaren [6] & & & $\begin{array}{l}\text { Moul et al. [15], } \\
\text { Madden et al. } \\
\text { [16], } \\
\text { Emery et al. [17], } \\
\text { Emery et al. [18]. }\end{array}$ \\
\hline & & & & \\
Colcombe [5] & & $\begin{array}{l}\text { Dustman et al. } \\
\text { [1], } \\
\text { Hill et al. [13], } \\
\text { Perri et al. [14], } \\
\text { Hassmen et al. } \\
\text { [21], } \\
\text { Bary et al. [19], } \\
\text { Powell et al. [20]. }\end{array}$ & $\begin{array}{l}\text { Madden et al. [16], } \\
\text { Emery et al. [18], } \\
\text { Emery et al. [17]. }\end{array}$ & \\
& & & \\
& & & \\
\hline
\end{tabular}

\section{RESULTS}

Results from meta-analysis [3-6] showed tendency of some cognitive tests to be more sensitive in examining results of physical training. Gates [3] and Heyn [4] meta-analysis included participants with MCI and dementia. $\mathrm{MCI}$ as a group of clinical syndromes has multiple diagnostic criteria, therefore varied MMSE scores of participants included in metaanalyzes has been reported [3, 4]. Heterogeneity of results causes several difficulties in comparing of studies. Nevertheless, some tendencies of cognitive tests can be observed. Gates et al. [3] reported statistically significant positive influence of aerobic training on verbal fluency tests only. Moreover, strength training improved performance in memory tests, however data were based on 2 interventions only [3].

Colcombe and Kramer [5] analyzed researches containing multi-modal training, one research based on MCI participants' results, another study contained intervention group suffering from depression. Angevaren [6] included studies with aerobic training and healthy elderly participants only, therefore comparing results from Colcombe and Kramer [5] and Angevaren [6] could be misleading.

Additionally, analyzed meta-analyzes in our paper contain 10 common studies. Consequently, direct results of studies [3-6] cannot be compared. However, some conclusions could be obtained. Meta-analysis [5] reported largest effect of multi-modal training (strength and aerobic) on cognitive functioning. Additionally, physical exercise influence on every cognitive tests category; executive and control type of tests results in largest effect size.

Angevaren [6] et al. showed largest effects of aerobic exercise on motor function and auditory attention (effect sizes of 1.17 and 0.50 , respectively), cognitive speed and visual attention resulted in less ES (both 0.26 , all ES were obtained in comparison aerobic exercise with 'no intervention', not included in our paper). However, comparing with no exercise do not exclude effects other than aerobic training per se. Nevertheless, comparison of aerobic exercise with 'any intervention' (strength training, flexibility training) can give ambiguous results. Strength training can influence cognitive functioning, however, results can differ from effects of aerobic training. Interestingly, comparison between aerobic exercise and strength training showed large effect $(E S=2.30)$ of strength training on executive functions. However, results were based on one study only.

\section{DISCUSSION AND CONCLUSION}

Cognitive tests commonly used in examining of physical training effects and demonstrated largest effect size are MMSE, and auditory attention test: digit span, digit span backward, digit span forward.

Noteworthy, Voss et al. [22] proposed hypothesis in which physical activity improves cognitive accuracy, not cognitive speed. Moreover, taking part in intervention group can improve cognitive functioning unlike influence of physical activity per se. One of the possible factors could be increment in social activity and new environment of elderly participants in intervention group (attending to gym, meeting coparticipants).

Colcombe et al. [5] reported that control group consisted of participants in age ranged from 55 to $65(\mathrm{~g}$ $=0.108, \mathrm{SE}=0.053, \mathrm{n}=23, \mathrm{p}<.05)$, and 66-70 $(\mathrm{g}=$ $0.258, S E=0.045, n=48, p<.05)$ noted larger performance improvement than 71-80 participants $(g=$ $0.076, S E=0.058, n=25$, n.s.).

Angevaren et al. [6] showed reduction of $\mathrm{VO}_{2} \max$ in all but two examined control (no training) groups. $\mathrm{VO}_{2} \max$ increase in controls were statistically nonsignificant. 


\section{REEFERENCES}

1. Dustman R.E., Ruhling R.O., Russell E.M. et al.: Aerobic exercise training and improved neuropsychological function of older individuals. Neurobiol Aging, 1984, 5:35-42.

2. Clarkson-Smith L., Hartley A.A.: Relationships between physical exercise and cognitive abilities in older adults. Psychol Aging, 1989, 4:183-9.

3. Gates N., Fiatarone Singh M.A., Sachdev PS et al:: The effect of exercise training on cognitive function in older adults with mild cognitive impairment: a metaanalysis of randomized controlled trials. Am J Geriatr Psychiatry, 2013, 21:1086-97.

4. Heyn P., Abreu B.C., Ottenbacher K.J.: The effects of exercise training on elderly persons with cognitive impairment and dementia: a meta-analysis. Arch Phys Med Rehabil, 2004, 85:1694-704.

5. Colcombe S., Kramer A.F.: Fitness effects on the cognitive function of older adults: A Meta-Analytic study. Psychol Sci, 2003, 14:125-130.

6. Angevaren M., Aufdemkampe G., Verhaar H.J.J. et al.: Physical activity and enhanced fitness to improve cognitive function in older people without known cognitive impairment. Cochrane database Syst Rev, 2008, :CD005381.

7. Gajos A., Kujawski S., Gajos M. et al:: Effect of physical activity on cognitive functions in elderly = Wpływ aktywności fizycznej na funkcje poznawcze w podeszłym wieku. J. Heal. Sci. , 4:2014;04(08):091100.

8. Kramer A.F., Hahn S., Cohen N.J. et al.: Ageing, fitness and neurocognitive function. Nature, 1999, 400:418-9.

9. Matthews F.E., Stephan B.C.M., McKeith I.G. et al.: Two-year progression from mild cognitive impairment to dementia: to what extent do different definitions agree? J Am Geriatr Soc, 2008, 56:1424-33.

10. Lezak M.D., Howieson D.B., Loring D.W.: Neuropsychological Assessment. Oxford University Press: 2004.

11. Kessels R.P.C., Aleman A., Verhagen W.I.M. et al.: Cognitive functioning after whiplash injury: A metaanalysis. J Int Neuropsychol Soc, 2000, 6:271-278.

12. Molloy D.W., Richardson L.D., Crilly R.G.: The effects of a three-month exercise programme on neuropsychological function in elderly institutionalized women: a randomized controlled trial. Age Ageing, 1988, 17:303-10.

13. Hill R.D., Storandt M., Malley M.: The impact of longterm exercise training on psychological function in older adults. J Gerontol, 1993, 48:P12-7.

14. Perri S., Templer D.I.: The effects of an aerobic exercise program on psychological variables in older adults. Int J Aging Hum Dev, 1984, 20:167-72.
15. Moul J., Goldman B., Warren B.: Physical activity and cognitive performance in the older population. $J$ aging ..., 1995.

16. Madden D.J., Blumenthal J.A., Allen P.A. et al.: Improving aerobic capacity in healthy older adults does not necessarily lead to improved cognitive performance. Psychol Aging, 1989, 4:307-20.

17. Emery C.F., Gatz M.: Psychological and cognitive effects of an exercise program for community-residing older adults. Gerontologist, 1990, 30:184-8.

18. Emery C.F., Schein R.L., Hauck E.R. et al.: Psychological and cognitive outcomes of a randomized trial of exercise among patients with chronic obstructive pulmonary disease. Health Psychol, 1998, 17:232-40.

19. Barry A.J., Steinmetz J.R., Page H.F. et al.: The effects of physical conditioning on older individuals. II. Motor performance and cognitive function. J Gerontol, 1966, 21:192-9.

20. Powell R.R.: Psychological effects of exercise therapy upon institutionalized geriatric mental patients. $J$ Gerontol, 1974, 29:157-61.

21. Hassmén P., Ceci R., Bäckman L.: Exercise for older women: a training method and its influences on physical and cognitive performance. Eur J Appl Physiol Occup Physiol, 1992, 64:460-466.

22. Voss M.W., Nagamatsu L.S., Liu-Ambrose T. et al.: Exercise, brain, and cognition across the life span. $J$ Appl Physiol, 2011, 111:1505-13.

Address for correspondence:

Department of Geriatrics

Nicolaus Copernicus University

Collegium Medicum in Bydgoszcz

ul. M. Curie Skłodowskiej 9, 85-094 Bydgoszcz

Szpital Uniwersytecki nr $1 \mathrm{im}$. dr. A. Jurasza, IX piętro tel. (52) 585-40-21

fax (52) 585-49-21

kikgeriat@cm.umk.pl

Agnieszka Gajos (e-mail: kikgeriat@cm.umk.pl)

Małgorzata Gajos (e-mail: kikgeriat@cm.umk.pl)

Wojciech Stemplowski (e-mail: kikgeriat@cm.umk.pl)

Natalia Ciesielska (e-mail: kikgeriat@cm.umk.pl)

Kornelia Kędziora-Kornatowska

(e-mail: kikgeriat@cm.umk.pl)

Received: 15.04.2015

Accepted for publication: 8.06.2015 